\title{
Using the Hot Embossing Technology for the Realization of Micro- technical Structures in Medical Imaging
}

\author{
Markus Detert ${ }^{1}$, Stefan Friesecke ${ }^{1}$, Martin Deckert ${ }^{1}$, Georg Rose ${ }^{2}$, Bertram Schmidt ${ }^{1}$, Mandy Kaiser ${ }^{2}$ \\ ${ }^{1}$ Institute of Micro and Sensor Systems, Otto-von-Guericke-University, Magdeburg, Germany, markus.detert@ovgu.de \\ ${ }^{2}$ Institute for Electronics, Signal Processing and Communications, Otto-von-Guericke-University, Magdeburg, Germany
}

\begin{abstract}
The technology of hot embossing is an established process for many years in the realization of electrical and optical conductors. The ability of this method is the realization of robust and reliable structures. In connection with the studies presented here, these skills are applied to the three-dimensional shape of a catheter or other instruments in the field of medical imaging. We generate as an example resonant circuits with the hot embossing technology. The use of hot-embossing technology improves the mechanical stability of the electrical conductor of the resonant circuits through the improved anchoring of the realized structures. The structural resolution is less than 100 microns and the structure height up to 1000 microns with very good reproducibility. The idea for the use of hot embossing is here pursued in two directions. Firstly, for the creation of channels that will later be functionalized by the addition of metal fillers to electrical conductors. For this solution we fill the embossed structures with polymer thick film paste materials. On the other hand, the known from the 3D-MID technology equipment will be used to emboss foil to create three-dimensional structures. As third way we use a classical FPC and laminate this to the surface of the three-dimensional shape with the help of hot embossing technology. All different solution ways will be characterized electrically with the needed values and their visibility in MR image process. The focus of the work, however, lies in the improvement of the mechanical properties of the resonant circuit. Due to the possibilities of the hot embossing technology we can transfer the ideas to other instruments and tools in the medical image processing. A final testing with accelerated temperature storage will be give a detailed answer to the mechanical behavior, because all tools are used under difficult conditions.
\end{abstract}

\section{$1 \quad$ Introduction for microtechnical structures in medical imaging}

MR imaging proves to be an eminently suitable modality for guiding minimal-invasive interventions due to its beneficial attributes such as superior soft tissue contrast, the possibility of arbitrary slice positioning and the absence of ionizing radiation. In contrast to typical close-bore MR scanners, open MR scanners - such as Philips Panorama HFO - provide an improved patient access. Within this MR environment interventions e.g. the placement of afterloading catheters for brachytherapy using an MRcompatible puncture needle, have been performed [1].

Instruments like catheters appear hypointense in the MR image due to their signal-loss artifact or can be even almost invisible. Therefore, suitable visualization techniques are required in order to highlight the instrument's tip and thus enable a precise positioning of the instrument to the area which need to be treated. Additionally, a robust tip tracking would allow an automatic slice positioning regarding real time MR sequences. It can be distinguished between negative and positive contrast modification.

A paramagnetic coating onto the catheter distorts the homogenous static B0-field locally and results in a negative contrast within the MR image [2]. Regarding the hypointense appearance of afterloading catheters within the MR image, this method won't benefit a tip tracking. Another method for catheter tracking is realized by using local magnetic fields generated by a DC fed conductor struc- tures [3]. A subtraction of MR images with and without the DC current yields to an image where the catheter can be seen clearly. The main drawback of this method consists in the risk of rf induced heating.

A method, which does not suffer from the mentioned deficiencies, is the usage of resonant circuits tuned to the Larmor frequency of the protons [4]. The Lamor frequency is defined by equation (1).

$$
\begin{aligned}
& f=\frac{\gamma}{2 \pi} \cdot B_{0} \\
& \frac{\gamma}{2 \pi}=42,58 \cdot \frac{M H z}{T}
\end{aligned}
$$

Equation (2) represents the gyromagnetic ratio for nuclei of hydrogen atoms. Hence, for a 1.0T MR scanner, the Larmor frequency amounts to $42.58 \mathrm{MHz}$. MR imaging is based on the deflection of nuclear spins from their equilibrium positions to the flip angle $\alpha$ due to the incident circular polarized B1-field at the Larmor frequency. During their return to the energy-efficient state an echo signal is measured. When a resonant circuit, tuned to the Larmor frequency, is used within the MR environment, an rf current is induced into the microcoil. This current generates a linear polarized magnetic field, which leads to a stronger excitation of the spins and results in local hyperintense signal intensity while using gradient echo sequences with a low flip angle. Among other aspects, the strength of the 
effect depends on the quality factor of the resonant circuit, the orientation of the coil referred to the B1-field and the adjusted flip angle. Within this paper various microsystems technologies for fabricating resonant markers will be compared in order to estimate their potential for a subsequent application.

The following requirements refer to the usage of a $6 \mathrm{~F}$ afterloading catheter for brachytherapy on which the resonant circuit shall be located at the distal end. According to the direction of the static magnetic B0-field and the procedure of the intervention, variant types of coils are particularly advantageous.

The dimensions of the resonant circuit must be kept as low as possible in order to prevent undesired effects such as property changes of the catheter. Therefore, the enlargement of the diameter need to be below $100 \mu \mathrm{m}$ and the maximum entire length of the resonant circuit is set to $20 \mathrm{~mm}$. The materials which are used must not cause any susceptibility artifacts. This can be ensured by process appropriate materials with an absolute value of the materials susceptibility compared to water below $10 \mathrm{ppm}$ (noticeable, but not significant image distortion), preferential below 3 ppm (no image abnormalities) [5].

In order to enable a precise tip tracking, the maximum size of the resulting hyperintensed area within the MR image is limited to $25 \mathrm{~mm}^{2}$. A quality factor of more than 20 is required to generate adequate local signal amplification. There must also be the possibility of a biocompatible housing for a subsequent clinical application.

\section{Realization of test structures and built-up of test vehicles}

The used resonance marker structure is shown in Figure 1. For the built-up of test vehicles we use the original material of the standard catheters.
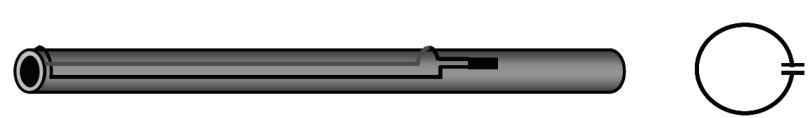

Image 1 Resonance marker structure, Source: [7]

The hot embossing technology is a very comfortable technique to realize structures for our resonance markers.

\begin{tabular}{|l|l|l|}
\hline Line width & Pitch & Height \\
\hline Down to $0,2 \mu \mathrm{m}$ & Down to $0,4 \mu \mathrm{m}$ & $\begin{array}{l}\text { Up to } 1 \ldots 1,4 \mu \mathrm{m} \\
\text { (at }<1 \mu \mathrm{m} \text { width) }\end{array}$ \\
\hline$>1 \mu \mathrm{m}$ & $>1 \mu \mathrm{m}$ & $\begin{array}{l}\text { Depends on resolu- } \\
\text { tion (up to } \\
500 \ldots 1000 \mu \mathrm{m})\end{array}$ \\
\hline
\end{tabular}

We follow in our strategy to different base type of the embossing technology. In the first one we use the classical technology with the attached metal foil (See Figure 2).

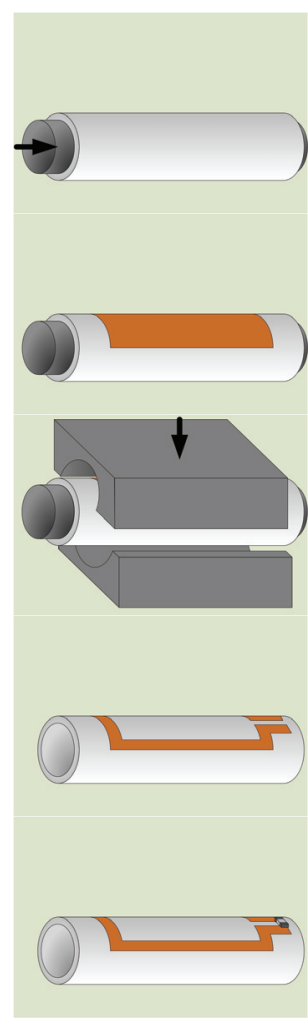

a) Inserting a core stabilization

b) Attaching the metal foil on the medical instrument

c) Punching out the tracks by a negative embossing stamp by pressure and temperature

d) Removal of the embossing punch

e) fine alignment with MRcompatible capacity

Image 2 Technology steps for the hot embossing process with a foil

The more interesting technology is the second way. There we use the hot embossing to make cavities at the catheter surface. In these cavities we bring later metallic particles with the polymer filled materials at the surface of the catheters.

For a better evaluation of our results we work in two phases. In phase 1 we work only at a two dimensional substrate. We make the test substrate with the same material like the original catheters (Figure 3). Only in the phase 2 we work then at the surface of the catheters directly.

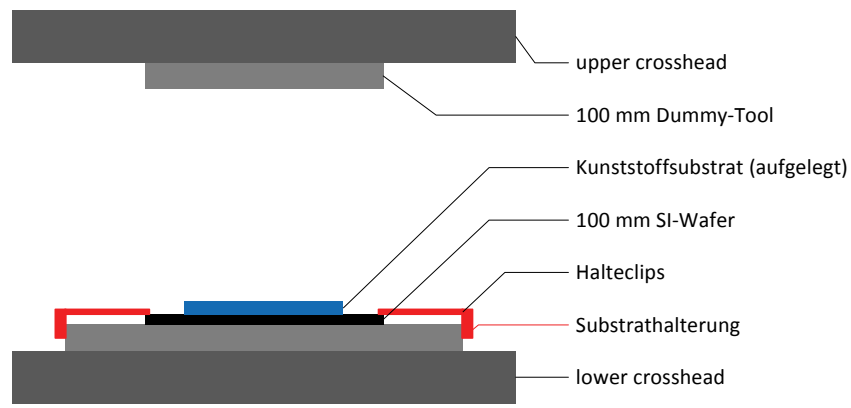

Image 3 Hot embossing at the $2 \mathrm{D}$ substrates, which are manufactures with the original catheter material

For the detailed analysis of our hot embossing investigations we used a standard technology print. We made the 
design on the background of our knowledge from other technologies. So we designed the layout of Figure 4, which was used for the phase 1 investigations.
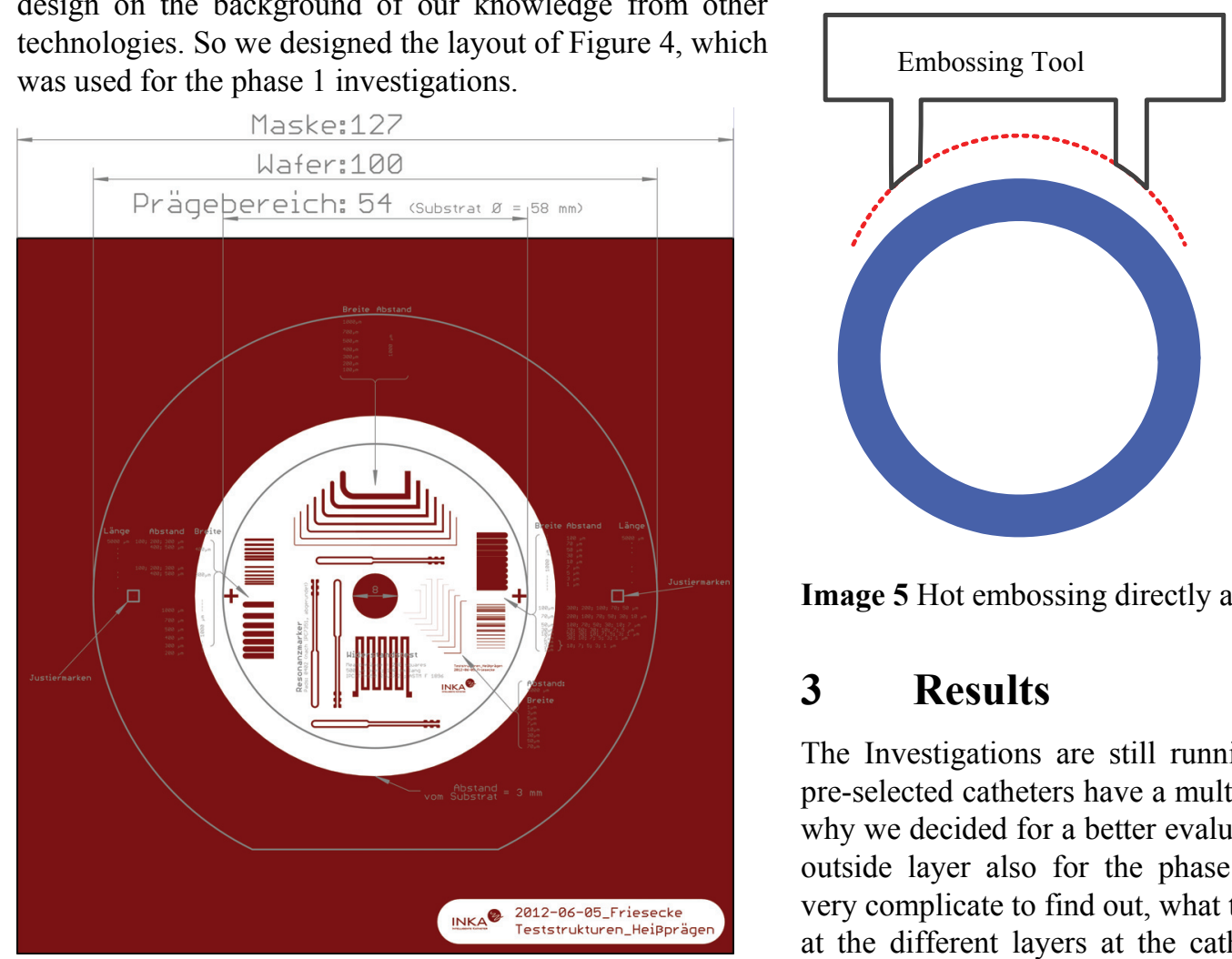

Image 5 Hot embossing directly at catheter surface

\section{Results}

The Investigations are still running at this time. All the pre-selected catheters have a multilayer technology. That's why we decided for a better evaluation, that we needed the outside layer also for the phase 1 investigations. It was very complicate to find out, what type of material was used at the different layers at the catheters from the different

Image 4 Layout for the realization of the phase 1 structures at $2 \mathrm{D}$ substrates

manufacturers. Table 1 illustrates the current level in our qualification process, which is still running.

During the next 6 weeks we expect some additional results

For the directly hot embossing at the catheter surface we need a convex stamp tool. At this time we build this tool for the phase 2 experiments. Figure 5 shows the situation with the $3 \mathrm{D}$ tool.

from our test vehicles in the different characterization processes. So we will give some more details during the poster session at the conference.

\begin{tabular}{|c|c|c|c|c|c|c|c|}
\hline & $\begin{array}{l}\text { Hot embossing } \\
\text { foil type }\end{array}$ & $\begin{array}{l}\text { Hot embos- } \\
\text { sing } \\
\text { paste type }\end{array}$ & $\begin{array}{l}\text { Wound } \\
\text { wire }\end{array}$ & $\begin{array}{c}\text { Aerosol } \\
\text { deposition }\end{array}$ & LTCC & FPC & $\begin{array}{l}\text { Thin film } \\
\text { technology }\end{array}$ \\
\hline Q-factor & + & $\mathrm{O}$ & ++ & $\mathrm{O}$ & + & $\mathrm{O}$ & $\mathrm{O}$ \\
\hline tunability & $\mathrm{O}$ & $\mathrm{O}$ & ++ & $\mathrm{O}$ & + & + & + \\
\hline reproducibility & + & $\mathrm{O}$ & - & - & ++ & ++ & + \\
\hline simple fabrication & + & + & ++ & + & - & $\mathrm{O}$ & - \\
\hline mechanically robust & + & + & ++ & - & + & + & + \\
\hline $\begin{array}{l}\text { negligible increase } \\
\text { of diameter }\end{array}$ & ++ & ++ & - & ++ & - & + & + \\
\hline $\begin{array}{l}\text { possibility to in- } \\
\text { tegrate capacity }\end{array}$ & - & - & - & + & + & + & + \\
\hline $\begin{array}{l}\text { not influenced by } \\
\text { catheter material }\end{array}$ & -- & -- & + & $\mathrm{O}$ & + & + & + \\
\hline $\begin{array}{l}\text { attachment to cathe- } \\
\text { ter }\end{array}$ & ++ & ++ & $\mathrm{O}$ & + & - & - & - \\
\hline structural resolution & $\mathrm{O}$ & ++ & -- & + & ++ & ++ & ++ \\
\hline
\end{tabular}

$++=\operatorname{very} \operatorname{good} /+=\operatorname{good} / \mathrm{o}=$ neutral $/ \mathrm{-}=\mathrm{bad} /--=$ very bad 


\section{Conclusions}

During the experimental work it was obtained, that the high standards for manufacturing processes of the micro system technology will implement the medical requirements at the needed level.

An important aspect is the knowledge gained to other work at the department of microsystems technology. So we can prove with the previous experiments, that very small structures in the critical aspect-ratio-ratios can be realized.

After completion of function tests these markers can be evaluated for their suitability under MR conditions in detail. The goal to realize structures down to a width of $10 \mu \mathrm{m}$ and a height of $100 \mu \mathrm{m}$ will be the most urgent action in the steps of these investigations. The last step will be the qualifying with the accelerated test methods. Then will be seen what kind of manufacturing technology provides the ideal result for the resonant marker. Finally it depends from the level of costs during the manufacturing processes and the reachable level of function quality.

\section{References}

[1] Ricke, Jens; Thormann, Markus; Ludewig, Matthias; Jungnickel, Kerstin; Grosser, Oliver; Wybranski, Christian et al. (2010): MR-guided liver tumor ablation employing open high-field 1.0T MRI for imageguided brachytherapy. In: Eur Radiol 20 (8), S. 1985

[2] Bakker, Chris J.G; Hoogeveen, Romhild M.; Weber, Jan; van Vaals, Joop J.; Viergever, Max A.; Mali, Willem P. (1996): Visualization of dedicated catheters using fast scanning techniques with potential for MRguided vascular interventions. In: Magn. Reson. Med 36 (6), S. 816-820.

[3] Glowinski, Arndt; Kursch, J.; Adam, Gerhard; Bücker, Arno; Noll, T. G.; Günther, Rolf W. (1998): Device visualization for interventional MRI using local magnetic fields: basic theory and its application to catheter visualization. In: Medical Imaging, IEEE Transactions on 17 (5), S. 786-793.

[4] Burl, Michael; Coutts, Glyn A.; Young, Ian R. (1996): Tuned fiducial markers to identify body locations with minimal perturbation of tissue magnetization. In: Magn. Reson. Med 36 (3), S. 491-493.

[5] Schenck, J. F. (1996): The role of magnetic susceptibility in magnetic resonance imaging: MRI magnetic compatibility of the first and second kinds. In: Med Phys 23 (6), S. 815-850.
[6] Nopper, R.; Niekrawietz, R.; Reindl, L. (2010): Wireless Readout of Passive LC Sensors. In: IEEE Trans. Instrum. Meas 59 (9), S. 2450-2457.

[7] Quick, H. ; Zenge, M. ; Kuehl, H. ; Kaiser, G. ; Eggebrecht, H. ; Aker, S. ; Massing, S. ; Ladd, M.: Wireless Active Catheter Visualization: Passive Decoupling Methods and Their Impact on Catheter Visibility. In: Proc. Intl. Soc. Mag. Reson. Med (2005), Nr. 13, S. 2163-2164.

[8] Kaiser, M.; Detert, M.; Schmidt B.; Rose, G. (2012): Technology Matrix: Production technologies of miniature instrument resonance markers for visualization under MRI, Congress MedTech Pharma 2012, Postersession 